\title{
Leitthema
}

Bundesgesundheitsbl 2015 $\cdot 58: 533-542$

DOI 10.1007/s00103-015-2146-1

Online publiziert: 21. April 2015

(c) Springer-Verlag Berlin Heidelberg 2015

Anke-Christine Saß ${ }^{1}$ B. Grüne ${ }^{2,3} \cdot$ A.-K. Brettschneider ${ }^{1}$ A. Rommel ${ }^{1}$ O. Razum ${ }^{3}$. U. Ellert ${ }^{1}$

${ }^{1}$ Abteilung für Epidemiologie und Gesundheitsmonitoring, Robert Koch-Institut, Berlin, Deutschland

2 Institut für Therapieforschung, München, Deutschland

${ }^{3}$ Fakultät für Gesundheitswissenschaften, Universität Bielefeld, Bielefeld, Deutschland

\section{Beteiligung von Menschen mit Migrationshintergrund an Gesundheitssurveys des Robert Koch-Instituts}

Menschen mit Migrationshintergrund sind in Deutschland und auch in zahlreichen anderen Ländern Westeuropas eine große und wachsende Bevölkerungsgruppe. Im Jahr 2013 hatten 20,5\% der Menschen in Deutschland einen Migrationshintergrund [1], das heißt, sie selbst oder mindestens ein Elternteil sind nach Deutschland zugewandert. Seit vielen Jahren sind die Lebensbedingungen und die Gesundheit von Menschen mit Migrationshintergrund in Deutschland ein wichtiges Thema in der wissenschaftlichen Forschung. Hieraus ergeben sich Hinweise für die Politik und - aus Politik und Praxis - auch neue Forschungsfragen. Menschen mit Migrationshintergrund unterscheiden sich in vielen Bereichen des Lebens, unter anderem in ihrer Gesundheit, von den Menschen im Zielland der Migration. Die Befundlage ist jedoch uneinheitlich: Menschen mit Migrationshintergrund sind nicht generell gesünder oder kränker als Personen ohne Migrationshintergrund und weisen spezifische Risiken und Ressourcen auf [24]. Protektiv können zum Beispiel soziale Netzwerke wirken, die nach Deutschland zugewanderte Menschen hier aufbauen. Ein erhöhtes Risiko kann für bestimmte Infektionskrankheiten bestehen, unter anderem für Tuberkulose. Gesundheitschancen und Krankheitsrisiken von Menschen mit Migrationshintergrund differieren nach Herkunftsland und Migrationsgeneration (selbst zugewandert oder in Deutschland geboren), aber auch nach Alter und Geschlecht. Außerdem zeigt sich bei den Gesundheitsoutcomes von Migrantinnen und Migranten die enge Verknüpfung von Gesundheit und sozialer Lage deutlich und überlagert den Zusammenhang zwischen Migrationshintergrund und Gesundheit.

Angesichts der quantitativen Bedeutung der Personengruppe mit Migrationshintergrund müsste ein kontinuierliches Monitoring der Gesundheit dieser Bevölkerungsgruppe etabliert sein. Dies ist für die Weiterentwicklung von Maßnahmen der Gesundheitsförderung und Prävention von Bedeutung, ebenso für kurative, rehabilitative und pflegerische Angebote des Gesundheitssystems [5]. Tatsächlich aber ist die Datenlage zur Gesundheit von Menschen mit Migrationshintergrund in Deutschland nach wie vor unzureichend. In den wenigen vorliegenden Erhebungen wird der Migrationshintergrund oft nur unvollständig erfasst, Angaben zum sozioökonomischen Status fehlen (zum Beispiel amtliche Statistik, siehe [3]). Studien können diese Daten erheben, sie konzentrieren sich aber meist auf bestimmte Regionen, Personengruppen oder bestimmte Erkrankungen [6-8].

Die Teilnahme von Menschen mit Migrationshintergrund an Studien erweist sich oft als schwierig. Zunächst ist die Identifizierung der Zielpersonen problematisch. Die komplexe Definition „Migrationshintergrund" lässt sich nicht an einem bei den Einwohnermeldeämtern registrierten Merkmal festmachen, wie zum Beispiel „keine deutsche Staatsangehörigkeit“. Deshalb kommen bei Studien, die sich ausschließlich an Menschen mit Migrationshintergrund richten, verschiedene Verfahren zum Einsatz, um die Zielpersonen zu identifizieren, zum Beispiel Namensalgorithmen [9]. Für einige große Gruppen von Migrantinnen und $\mathrm{Mi}$ granten, zum Beispiel Personen, die als Spätaussiedler aus der ehemaligen Sowjetunion oder anderen osteuropäischen Staaten nach Deutschland gekommen sind, ist dieses Verfahren jedoch nicht anwendbar. Ihre Namen sind von denen der Personen ohne Migrationshintergrund oft nicht zu unterscheiden. Wenn die Zielpersonen identifiziert wurden, erweist es sich häufig als schwierig, sie für eine Teilnahme zu gewinnen. Diese Problematik besteht auch bei bevölkerungsbezogenen Gesundheitsstudien, die sich an die gesamte Bevölkerung richten und die Teilnehmenden, zunächst ohne Informationen über ihren Migrationshintergrund, per Zufallsstichprobe aus Einwohnermelderegistern ziehen. Menschen mit Migrationshintergrund haben für die Teilnahme an Gesundheitsstudien andere Barrieren zu überwinden als Menschen ohne Migrationshintergrund [10]. Hierzu zählen insbesondere sprachliche Barrieren, kulturelle Werte und Normen sowie mangelhafte Erreichbarkeit $[6,10,11]$.

Zwei aktuelle bevölkerungsbezogene Gesundheitsstudien in Deutschland haben Menschen mit Migrationshintergrund einbezogen: die „Studie zur Gesundheit Erwachsener in Deutschland“ (DEGS 1, 2008-2011) sowie die „Studie zur Gesundheit von Kindern und Ju- 
gendlichen" (KiGGS Welle 1, 2009-2012). Es wird hier analysiert, in welchem Umfang sich Kinder, Jugendliche und Erwachsene mit Migrationshintergrund an den Studien beteiligt haben. Anschließend wird untersucht, ob die Zusammensetzung der Teilnehmenden mit Migrationshintergrund hinsichtlich Alter, Geschlecht und weiterer soziodemografischer Variablen der Bevölkerung mit Migrationshintergrund in Deutschland entspricht. In einem dritten Schritt wird exemplarisch geprüft, wie sich die Zusammensetzung der Stichproben von Erwachsenen mit Migrationshintergrund auf die Ausprägung ausgewählter Gesundheitsvariablen auswirkt. Auf Basis der Analysen werden die Empfehlungen für die Auswertungen der Gesundheitsdaten von Menschen mit Migrationshintergrund in beiden Studien abgeleitet und Aussagen über Rekrutierungsstrategien für diese Bevölkerungsgruppe getroffen.

\section{Material und Methoden}

\section{Design und Stichproben}

Die „Studie zur Gesundheit Erwachsener in Deutschland“ (DEGS ) und die „Studie zur Gesundheit von Kindern und Jugendlichen in Deutschland" (KiGGS) sind Bestandteile des Gesundheitsmonitorings des Robert Koch-Instituts (RKI). Konzept und Design der Studien sind an anderer Stelle ausführlich beschrieben (DEGS: [12-16] und KiGGS: [17-19]).

Zielpopulation von DEGS ist die in Deutschland lebende Bevölkerung im Alter von 18 bis 79 Jahren. Die erste Erhebungswelle (DEGS 1) wurde von 2008 bis 2011 durchgeführt und umfasste Befragungen, Untersuchungen und Tests [20, 21]. An DEGS 1 beteiligten sich sowohl ehemalige Teilnehmerinnen und Teilnehmer des Bundes-Gesundheitssurveys 1998 (BGS 98; Längsschnitt) als auch neu gezogene Personen (Einwohnermeldeamtsstichprobe). Aufgrund dieses Mischdesigns sind quer- und längsschnittliche Analysen möglich. Insgesamt nahmen 8152 Personen an DEGS 1 teil, darunter 4193 Ersteingeladene (Response $42 \%$ ) und 3959 ehemalige Teilnehmerinnen und Teilnehmer des BGS 98 (Response $62 \%$ ).
KiGGS Welle 1 (2009-2012) richtete sich an Kinder, Jugendliche und junge Erwachsene. Mit den Eltern der 0- bis 17-Jährigen wurden Telefoninterviews geführt. $\mathrm{Ab}$ einem Alter von 11 Jahren wurden die Jugendlichen auch selbst telefonisch zu ihrer Gesundheit befragt. Die Stichprobe von KiGGS Welle 1 bestand zum einen aus den Teilnehmenden der KiGGS-Basiserhebung (2003-2006), die inzwischen 6 bis 24 Jahre alt waren (Längsschnitt). Eine neue Querschnittstichprobe 0- bis 6-Jähriger wurde zufällig aus den Melderegistern der ursprünglichen 167 Studienorte gezogen. Insgesamt nahmen 12.368 Kinder und Jugendliche in dem für den Querschnitt relevanten Altersbereich von 0 bis 17 Jahren an KiGGS Welle 1 teil. Darunter waren 4455 Ersteingeladene (Response $38,8 \%$ ) und 7913 Wiedereingeladene (Response 72,9\%). Die nachfolgenden Ergebnisse beziehen sich auf die 0 - bis 17 -jährigen Kinder und Jugendlichen.

\section{Einbeziehung von Menschen mit Migrationshinter- grund-Maßnahmen}

Um Verzerrungen in der Realisierung der Stichproben entgegenzuwirken, wurde in DEGS 1 ein Oversampling von Personen mit nicht-deutscher Staatsangehörigkeit durchgeführt [13, 22, 23]. Die DEGS 1-Gesundheitsfragebögen und Einverständniserklärungen wurden in Russisch, Türkisch, Serbokroatisch und Englisch übersetzt [13]. Im Bundes-Gesundheitssurvey 1998 (BGS 98) gab es weder ein Oversampling noch übersetzte Fragebögen. Ausreichende Deutschkenntnisse waren eine Teilnahmevoraussetzung [24].

Auch in der KiGGS-Basiserhebung und für die neu gezogene Stichprobe 0bis 6-Jähriger in KiGGS Welle 1 wurde ein Oversampling von Personen mit nichtdeutscher Staatsangehörigkeit durchgeführt $[6,13,22,23]$. Die ersteingeladenen Teilnehmenden mit Migrationshintergrund wurden in beiden Studien computergestützt anhand ihres Vor- und Nachnamens einer Sprachgruppe zugeordnet und erhielten übersetzte Einladungsschreiben (Türkisch, Russisch, Serbokroatisch, Arabisch, Englisch, Vietnamesisch). Außerdem gab es als Alternative zum Telefoninterview übersetzte schrift- liche Fragebögen. Darüber hinaus hatte es in der KiGGS-Basiserhebung umfassende Aktivitäten zur Einbindung von Familien mit Migrationshintergrund gegeben, unter anderem eine migrantenspezifische Öffentlichkeitsarbeit (Migrantenmedien etc.) sowie ein interkulturell geschultes Feldteam [25]. Dies konnte bei der telefonischen Wiederholungsbefragung nicht in gleichem Umfang realisiert werden.

\section{Einbeziehung von Menschen mit Migrationshintergrund - Methodik der Überprüfung}

Die Beteiligung von Menschen mit Migrationshintergrund an den Gesundheitssurveys des Robert Koch-Instituts wurde anhand des Mikrozensus überprüft. Dafür wurde die Verteilung ausgewählter soziodemografischer und Gesundheitsvariablen in den Stichproben von DEGS 1 bzw. KiGGS Welle 1 mit der Verteilung im Mikrozensus verglichen. Der Mikrozensus, eine repräsentative Haushaltsbefragung der amtlichen Statistik Deutschlands [26] ist hierfür die geeignete Datenquelle, weil er die Bevölkerung mit Migrationshintergrund in Deutschland mit einschließt und die Teilnahme am Mikrozensus verpflichtend ist. Jährlich wird ein Prozent der Privathaushalte stellvertretend für die Gesamtbevölkerung Deutschlands zu ihren Lebensbedingungen befragt [26, 27]. Der Mikrozensus erhebt umfangreiche Informationen zur wirtschaftlichen und sozialen Lage der Haushalte, zu Erwerbstätigkeit, Arbeitsuche, Wohnverhältnissen und seit 2005 auch zum Migrationshintergrund. Alle vier Jahre enthält das Befragungsprogramm einige Fragen zur Gesundheit. Für das Kindes- und Jugendalter sind die im Mikrozensus erhobenen Variablen zur Gesundheit (z. B. Schwerbehinderung, Rauchstatus) allerdings weniger gut geeignet. Spezielle Fragen zur Gesundheit von Kindern und Jugendlichen werden im Mikrozensus nicht gestellt.

Um Daten von Menschen mit Migrationshintergrund aus DEGS 1 bzw. KiGGS Welle 1 und dem Mikrozensus 2009 vergleichen zu können, wurde sichergestellt, dass alle für den Datenvergleich verwendeten Variablen in beiden Datensätzen gleich definiert werden. Dies gilt zunächst für den Migrationshintergrund, der in 
den Surveys und im Mikrozensus leicht unterschiedlich definiert wird. Um eine identische Datengrundlage zu schaffen, wurde vom Statistischen Bundesamt eine Sonderauswertung der Daten des Mikrozensus 2009 unter Anwendung der Definition des Migrationshintergrunds aus DEGS 1 und KiGGS Welle 1 vorgenommen (Definitionen siehe $\bullet$ Übersicht 1 ). Alle für den Vergleich herangezogenen soziodemografischen und gesundheitsbezogenen Variablen aus den Surveys wurden mit den entsprechenden Variablen aus dem Mikrozensus abgeglichen, ggf. erfolgte eine Umkodierung.

\section{Statistische Analysen}

Im Rahmen der statistischen Auswertung wurden Anteilswerte für Teilnehmende mit Migrationshintergrund berechnet und mit den Angaben aus dem Mikrozensus 2009 verglichen. Für die Erwachsenen (DEGS 1) wurden zahlreiche soziodemografische Variablen überprüft [28]. Im Folgenden werden die Ergebnisse bezüglich Geschlecht, Alter, Bildung, Migrationsgeneration und Staatsangehörigkeit berichtet. Eine Differenzierung nach einseitigem vs. beidseitigem Migrationshintergrund wurde - analog zu den vom Mikrozensus veröffentlichten Ergebnissen nicht vorgenommen. Analysen zum Einfluss dieses Merkmals im Erwachsenenalter stehen noch aus.

Für Kinder und Jugendliche wurden die Variablen Alter, Bildung der Eltern und einseitiger vs. beidseitiger Migrationshintergrund herangezogen. Die Unterscheidung nach einseitigem vs. beidseitigem Migrationshintergrund hatte sich in der KiGGS-Basiserhebung als wichtiges Differenzierungsmerkmal bei den Gesundheitsoutcomes erwiesen [4]. Der Vergleich erfolgte zwischen KiGGS Welle 1 (gesamte Stichprobe und Querschnitt- vs. Längsschnittstichprobe), der KiGGS-Basisuntersuchung und dem Mikrozensus 2009. Die KiGGS-Basisuntersuchung wurde in den Vergleich einbezogen, weil es dabei gelungen war, Familien mit Migrationshintergrund entsprechend ihrem Anteil an der Bevölkerung für die Teilnahme zu gewinnen [7]. Außerdem wurden für die Erwachsenen [28] ausgewählte Angaben zu Gesundheitsverhalten

Bundesgesundheitsbl 2015 · 58:533-542 DOI 10.1007/s00103-015-2146-1

(c) Springer-Verlag Berlin Heidelberg 2015

\section{A.-C. Saß · B. Grüne · A.-K. Brettschneider · A. Rommel • O. Razum • U. Ellert} Beteiligung von Menschen mit Migrationshintergrund
an Gesundheitssurveys des Robert Koch-Instituts

\section{Zusammenfassung}

Menschen mit Migrationshintergrund (MMH) sind eine große Bevölkerungsgruppe mit spezifischen Gesundheitschancen und Risiken. Daten zur gesundheitlichen Lage von MMH liegen nur in begrenztem Umfang vor, u. a. weil ihre Einbeziehung in Studien (z. B. aufgrund von Sprachbarrieren) schwierig ist. In der vorliegenden Arbeit wurde geprüft, inwieweit es in den bevölkerungsbasierten $\mathrm{Ge}$ sundheitssurveys des Robert Koch-Instituts gelungen ist, $\mathrm{MMH}$ in repräsentativem Umfang einzubeziehen. Daraus sollen Empfehlungen für Auswertungsmöglichkeiten und für die zukünftige Rekrutierung von $\mathrm{MMH}$ in Studien abgeleitet werden. Der Mikrozensus 2009 (MZ) des Statistischen Bundesamtes wurde herangezogen, um die Zusammensetzung der Stichprobe von MMH in der Studie zur Gesundheit von Kindern und Jugendlichen (KiGGS Welle 1, 2009-2012) und in der Studie zur Gesundheit Erwachsener in Deutschland (DEGS 1, 2008-2011) auf Repräsentativität in Bezug auf soziodemografische und migrationsspezifische Variablen zu prüfen. An DEGS1 haben 1107 MMH teilgenommen. Im Vergleich zum MZ sind be- stimmte Subgruppen in der Stichprobe unterrepräsentiert: selbst zugewanderte Menschen (1. Generation), Personen mit niedriger Bildung und türkische Staatsangehörige. Einige Altersgruppen sind hingegen überrepräsentiert. An KiGGS Welle 1 nahmen 2021 Kinder und Jugendliche mit MH teil. Es zeigte sich eine schlechtere Teilnahme bei niedrigem Bildungsniveau der Eltern. Die Beteiligung war bei Migranten insgesamt geringer als in der KiGGS-Basiserhebung. Die Daten der MMH aus DEGS1 und KiGGS Welle 1 sind für Auswertungen zur Gesundheit dieser Bevölkerungsgruppe geeignet. Analysen sollten jedoch nach Merkmalen wie Migrationsgeneration, Alter oder Bildung stratifiziert bzw. in statistischen Modellen adjustiert werden. Für eine repräsentative Einbeziehung von $\mathrm{MMH}$ in Surveys sind, speziell für Subgruppen, spezifische Maßnahmen der Teilnahmemotivation angeraten.

\section{Schlüsselwörter \\ Migration - Survey - Gesundheit . \\ Repräsentativität · Response}

\section{Participation of people with migration background in health surveys of the Robert Koch Institute}

\section{Abstract}

People with migration background (PMB) make up a huge section of the population with specific health chances and risks. There are only limited data available on the health situation of PMB, since inclusion of PMB in surveys is hindered, e.g. due to language barriers. The present study has examined to what extent the population-based health surveys of the Robert Koch Institute have managed to include a representative extent of PMB, with the aim of deriving recommendations for analysis options and future recruitment strategies. The $2009 \mathrm{mi}-$ crocensus (MC) of the Federal Statistical Office was used as the basis to check whether the sample of KiGGS Wave 1 (2009-2012) and DEGS 1 (2008-2011) are representative regarding socio-demographic and migrantspecific characteristics. 1107 PMB participated in DEGS 1. In comparison to the MC, particular sub-groups are underrepresented in the sample: people who immigrated themselves (first-generation migrants), people with a low education and Turkish citizens. On the other hand, some age groups are overrepresented. In KiGGS Wave 1, 2021 children and adolescents with a migration background participated. Response was lower if parents had a low education. In total, the participation of children and adolescents with a migration background was lower in comparison to the KiGGS baseline survey. The data on PMB in DEGS 1 and KiGGS Wave 1 are appropriate for health analyses of this population group. However, analyses should be stratified according to characteristics like migrant generation, age or education level, or these characteristics should be adjusted for in statistical models. In order to achieve a representative inclusion of people with a migration background, in future surveys sub-group-specific activities to increase participation of PMB are recommended.

\section{Keywords}

Migration · Survey $\cdot$ Health .

Representativeness $\cdot$ Response rate 


\begin{tabular}{l}
\hline Übersicht 1 Definition: Men- \\
schen mit Migrationshintergrund \\
in DEGS 1 und KiGGS Welle 1 \\
\hline In DEGS 1 wurden diejenigen als Personen \\
mit beidseitigem Migrationshintergrund de- \\
finiert, die selbst aus einem anderen Land zu- \\
gewandert sind und von denen mindestens \\
ein Elternteil nicht in Deutschland geboren \\
ist oder von denen beide Eltern zugewandert \\
sind. Als Personen mit einseitigem Migra- \\
tionshintergrund wurden diejenigen Perso- \\
nen definiert, die in Deutschland geboren \\
sind und von denen ein Elternteil aus einem \\
anderen Land zugewandert ist. Personen, die \\
bis einschließlich 1949 als Deutsche(r) ohne \\
Einbürgerung auf das heutige Bundesgebiet \\
zugewandert sind, werden nicht zu den Men- \\
schen mit Migrationshintergrund gerechnet. \\
In KiGGS Welle 1 wurden analog zur KiGGS- \\
Basiserhebung diejenigen als Personen mit \\
beidseitigem Migrationshintergrund definiert, \\
die selbst aus einem anderen Land zuge- \\
wandert sind und von denen mindestens ein \\
Elternteil nicht in Deutschland geboren ist \\
oder von denen beide Eltern zugewandert \\
oder nicht deutscher Staatsangehörigkeit sind. \\
Als Kinder und Jugendliche mit einseitigem \\
Migrationshintergrund wurden Kinder und \\
Jugendliche definiert, die in Deutschland ge- \\
boren sind und von denen ein Elternteil aus \\
einem anderen Land zugewandert und/oder \\
nichtdeutscher Staatsangehörigkeit ist [25]. \\
\hline
\end{tabular}

und Gesundheitszustand aus den Surveys mit den Daten aus dem Mikrozensus verglichen.

Die für DEGS 1 und für KiGGS Welle 1 entwickelten Gewichtungsfaktoren korrigieren Abweichungen der Stichprobe von der Bevölkerungsstruktur (Stand: 31.12.2010) bezüglich Alter, Geschlecht, Bildung, Staatsangehörigkeit, Region und Gemeindetyp [13, 22]. Sie gleichen auch die unterschiedliche Wiederteilnahmebereitschaft der Teilnehmenden des BGS 98 bzw. der KiGGS-Basiserhebung aus. Für altersstandardisierte Vergleiche der KiGGS-Basiserhebung mit KiGGS Welle 1 wurden die Daten der Basiserhebung bezüglich der oben genannten Merkmale neu gewichtet und auf den Bevölkerungsstand zum 31.12.2010 altersstandardisiert [22]. Neben den Datenanalysen mit den Gewichtungsfaktoren wurden auch ungewichtete Prävalenzen ermittelt, um den Einfluss der Gewichtung auf die Zusammensetzung der Stichprobe von Menschen mit Migrationshintergrund abschätzen zu können. Außerdem wurden
95\%-Konfidenzintervalle (KI) berechnet. $95 \%$-Konfidenzintervalle, die sich nicht überschneiden, wurden als statistisch signifikant gewertet. Aufgrund der Größe des Mikrozensus wurden bei den durchgeführten Tests Gruppenunterschiede mit p-Werten kleiner 0,01 als statistisch signifikant gewertet. Um den Gewichtungsfaktor miteinzubeziehen, wurden alle Analysen mit dem Verfahren für komplexe Stichproben von SPSS-20 durchgeführt.

\section{Ergebnisse}

\section{Teilnehmende mit Migrati- onshintergrund in DEGS 1 vs. Mikrozensus 2009}

An DEGS 1 haben sich 1107 Studienteilnehmerinnen und Studienteilnehmer mit Migrationshintergrund beteiligt. Das entspricht einem gewichteten Anteil von 19,8\% (ungewichtet: 14,2\%) der Gesamtstichprobe. Im Mikrozensus 2009 haben unter Anwendung der Definition des Migrationshintergrunds nach DEGS 1 20,5\% der Erwachsenen einen Migrationshintergrund.

Hinsichtlich der Geschlechtsverteilung bestehen keine Unterschiede zwischen Menschen mit Migrationshintergrund in DEGS 1 und dem Mikrozensus 2009. In DEGS 1 beträgt der Anteil der Frauen mit Migrationshintergrund 53,1\% (ungewichtet) bzw. 51,1\% (gewichtet) und der Anteil der Männer mit Migrationshintergrund 46,9\% (ungewichtet) bzw. 48,9\% (gewichtet). Im Vergleich dazu sind im Mikrozensus 2009 50,4\% der Personen mit Migrationshintergrund weiblich und 49,6\% männlich.

In $\bullet$ Tab. 1 sind die Häufigkeitsverteilungen von Frauen und Männern mit Migrationshintergrund in DEGS 1 und dem Mikrozensus 2009 nach soziodemografischen Merkmalen dargestellt. In den ungewichteten Häufigkeiten bestehen signifikante Unterschiede hinsichtlich des Alters. Ältere Frauen und Männer mit Migrationshintergrund (60-69 Jahre und 7079 Jahre) haben sich überproportional an DEGS 1 beteiligt. In den mittleren Altersgruppen ist zum Teil eine geringere Beteiligung zu beobachten (30- bis 49-jährige Männer). In den gewichteten Daten bestehen signifikante Unterschiede hin- sichtlich des Alters bei Männern mit Migrationshintergrund, unter anderem weil der Anteil der Männer in der jüngsten Altersgruppe durch die Gewichtung überproportional erhöht wird. Bei Frauen mit Migrationshintergrund werden die Abweichungen durch die Gewichtung ausgeglichen (• Tab. 1).

Auch hinsichtlich des Merkmals Bildung unterscheiden sich Frauen und Männer mit Migrationshintergrund in DEGS 1 (ungewichtet) und im Mikrozensus 2009 signifikant (• Tab. 1). Während Frauen und Männer mit Migrationshintergrund und niedrigem Bildungsniveau in DEGS 1 im Vergleich zum Mikrozensus 2009 unterrepräsentiert sind, sind Frauen und Männer mit Migrationshintergrund und hohem Bildungsstatus überrepräsentiert. Dieses Teilnahmemuster zeigt sich auch bei der Gesamtstichprobe, deshalb wurde die Variable Bildung in die Gewichtung einbezogen [13]. Die bei Teilnehmenden mit Migrationshintergrund beobachteten Unterschiede bestehen nach der Gewichtung nicht mehr.

In den ungewichteten Daten zeigen sich signifikante Unterschiede hinsichtlich der Migrationsgeneration zwischen DEGS 1 und dem Mikrozensus 2009 (• Tab. 1). Frauen und Männer mit Migrationshintergrund der zweiten Generation sind in DEGS $1 \mathrm{im}$ Vergleich zum Mikrozensus 2009 überrepräsentiert. Die Unterscheide reduzieren sich durch die Datengewichtung in DEGS 1. Die Signifikanz der Unterschiede bleibt jedoch auch nach der Datengewichtung bestehen (• Tab. 1).

Bezogen auf die Staatsangehörigkeit gibt es in den ungewichteten Daten signifikante Unterschiede zwischen den Teilnehmenden in DEGS 1 und dem Mikrozensus 2009 (• Tab. 1). In DEGS 1 ist der Anteil der Frauen und Männer mit Migrationshintergrund, die die deutsche Staatsangehörigkeit besitzen, höher als im Mikrozensus. Eine türkische Staatsangehörigkeit besitzen hingegen weniger Frauen und Männer mit Migrationshintergrund in DEGS 1. Die Datengewichtung kompensiert diese Unterschiede weitgehend (Merkmal Staatsangehörigkeit wird berücksichtigt).

Im Anschluss an die Überprüfung soziodemografischer Variablen wurden die im Mikrozensus verfügbaren Variablen 


\begin{tabular}{|c|c|c|c|c|c|c|}
\hline & \multicolumn{3}{|c|}{ Frauen mit Migrationshintergrund } & \multicolumn{3}{|c|}{ Männer mit Migrationshintergrund } \\
\hline & DEGS1 $1_{\text {ungewichtet }}$ & DEGS1 $1_{\text {gewichtet }}$ & Mikrozensus 2009 & DEGS1 $1_{\text {ungewichtet }}$ & DEGS1 $1_{\text {gewichtet }}$ & Mikrozensus 2009 \\
\hline & $(n=588)$ & & $(n=6.570 .000)$ & $(n=519)$ & & $(n=6.460 .000)$ \\
\hline & $\%(95 \%-K I)$ & $\%(95 \%-K I)$ & $\%$ & $\%(95 \%-K I)$ & $\%(95 \%-K I)$ & $\%$ \\
\hline Alter & $(p=0,0009)^{*}$ & $(p=0,0297)$ & & $(p<0,0001)^{*}$ & $(p<0,0001)^{*}$ & \\
\hline 18-29 Jahre & $17,7(14,8-21,0)$ & $24,4(20,4-28,9)$ & 19,7 & $22,7(19,3-26,5)$ & $31,0(26,3-36,1)$ & 19,1 \\
\hline 30-39 Jahre & $17,9(15,0-21,2)$ & $20,7(16,6-25,6)$ & 22,7 & $15,6(12,7-19,0)$ & $18,6(14,1-24,1)$ & 22,0 \\
\hline 40-49 Jahre & $19,9(16,9-23,3)$ & $21,3(17,3-25,8)$ & 21,4 & $15,4(12,6-18,8)$ & $16,7(12,8-21,5)$ & 23,2 \\
\hline 50-59 Jahre & $20,1(17,0-23,5)$ & $16,0(12,6-20,0)$ & 17,5 & $17,5(14,5-21,1)$ & $15,1(12,1-18,8)$ & 17,0 \\
\hline 60-69 Jahre & $14,8(12,1-17,9)$ & $11,0(8,5-14,3)$ & 11,8 & $19,3(16,1-22,9)$ & $13,1(10,2-16,7)$ & 12,1 \\
\hline 70-79 Jahre & $9,7(7,5-12,4)$ & $6,6(4,6-9,4)$ & 7,0 & $9,4(7,2-12,3)$ & $5,5(3,5-8,4)$ & 6,7 \\
\hline Bildung $^{a}$ & $(p<0,0001)^{*}$ & $(p=0,0297)$ & & $(p<0,0001)^{*}$ & $(p=0,4466)$ & \\
\hline Niedrig & $24,5(21,2-28,1)$ & $35,1(30,6-40,0)$ & 37,6 & $22,4(19,0-26,2)$ & $30,8(25,5-36,6)$ & 30,1 \\
\hline Mittel & $46,9(42,9-51,0)$ & $43,4(38,8-48,1)$ & 44,4 & $42,4(38,2-46,7)$ & $46,5(41,0-52,2)$ & 48,5 \\
\hline Hoch & $28,6(25,1-32,4)$ & $21,5(17,5-26,0)$ & 18,0 & $35,2(31,2-39,4)$ & $22,7(18,7-27,2)$ & 21,4 \\
\hline Migrationsgeneration & $(p<0,0001)^{*}$ & $(p<0,0001)^{*}$ & & $(p<0,0001)^{*}$ & $(p<0,0001)^{*}$ & \\
\hline $\begin{array}{l}\text { 1. Generation (selbst zu- } \\
\text { gewandert) }\end{array}$ & $59,9(55,8-63,8)$ & $67,7(62,4-72,6)$ & 75,1 & $58,4(54,1-62,6)$ & $64,4(59,6-68,9)$ & 74,1 \\
\hline $\begin{array}{l}\text { 2. Generation (in } \\
\text { Deutschland geboren) }\end{array}$ & $40,1(36,2-44,2)$ & $32,3(27,4-37,6)$ & 24,9 & $41,6(37,4-45,9)$ & $35,6(31,1-40,4)$ & 25,9 \\
\hline Staatsangehörigkeit & $(p<0,0001)^{*}$ & $(p=0,2159)$ & & $(p<0,0001)^{*}$ & $(p=0,0075)^{*}$ & \\
\hline Türkisch & $5,4(3,9-7,6)$ & $8,4(5,6-12,4)$ & 9,3 & $5,4(3,8-7,7)$ & $9,1(5,9-13,9)$ & 10,2 \\
\hline $\begin{array}{l}\text { Griechisch, Italienisch, } \\
\text { Portugiesisch, Spanisch }\end{array}$ & $2,7(1,7-4,4)$ & $4,4(2,6-7,2)$ & 5,8 & $6,9(5,1-9,5)$ & $10,8(7,0-16,4)$ & 8,2 \\
\hline Polnisch & $2,4(1,4-4,0)$ & $3,6(2,0-6,3)$ & 2,9 & $0,4(0,1-1,5)$ & $0,9(0,2-3,9)$ & 1,8 \\
\hline $\begin{array}{l}\text { Staaten der ehemaligen } \\
\text { Sowjetunion }\end{array}$ & $3,1(1,9-4,8)$ & $4,2(2,5-6,9)$ & 4,6 & $1,5(0,8-3,1)$ & $1,6(0,7-3,4)$ & 3,0 \\
\hline $\begin{array}{l}\text { Staaten des ehemaligen } \\
\text { Jugoslawien }\end{array}$ & $3,7(2,5-5,6)$ & $6,4(4,1-9,7)$ & 6,1 & $4,4(3,0-6,6)$ & $7,2(4,8-10,8)$ & 6,1 \\
\hline Sonstige Länder & $11,7(9,4-14,6)$ & $16,3(12,6-20,9)$ & 14,2 & $10,4(8,1-13,4)$ & $14,7(10,6-19,9)$ & 14,6 \\
\hline Deutsch & $70,9(67,1-74,5)$ & $56,7(51,4-61,9)$ & 57,2 & $70,8(66,8-74,6)$ & $55,6(49,2-61,8)$ & 56,0 \\
\hline
\end{tabular}

zur Gesundheit herangezogen, um Hinweise auf Auswirkungen der Stichprobenzusammensetzung auf Analysen zur Gesundheit zu erhalten. Dazu wurden die gewichteten Daten aus DEGS 1 verwendet (• Tab. 2). Signifikante Unterschiede bestehen zwischen Frauen mit Migrationshintergrund in den beiden Erhebungen hinsichtlich des Rauchstatus und zwischen Männern hinsichtlich des (gruppierten) Body-Mass-Index (BMI). In DEGS 1 gibt es mehr Raucherinnen mit Migrationshintergrund (+ 8,6 Prozentpunkte) als im Mikrozensus 2009. Es haben weniger übergewichtige Männer mit Migrationshintergrund (-6,8 Prozentpunkte) und mehr adipöse Männer mit Migrationshintergrund (+ 7,5 Prozentpunkte) an DEGS 1 teilgenommen. Keine Häufigkeitsunterschiede bestehen bezüg- lich amtlich anerkannter Schwerbehinderungen (Grad der Behinderung $\geq 50$ ) in DEGS 1 und im Mikrozensus 2009.

\section{Teilnahme von Kindern und} Jugendlichen mit Migrationshintergrund an KiGGS Welle 1 vs. KiGGS-Basiserhebung und Mikrozensus 2009

An KiGGS Welle 1 haben sich insgesamt 1104 Kinder und Jugendliche mit beidseitigem Migrationshintergrund beteiligt. Das entspricht in der gewichteten Stichprobe 16,0\% der Teilnehmenden (KiGGS-Basiserhebung: 17,6\%) (• Tab. 3). Dem Mikrozensus 2009 zufolge entsprach der Anteil an Kindern und Jugendlichen mit beidseitigem Migrationshintergrund 18,1\%. Ein Vergleich der ungewichteten Stichproben der KiGGS-Basiserhebung und KiGGS Welle 1 zeigt jedoch, dass an KiGGS Welle 1 nur 8,9\% Kinder und Jugendliche mit beidseitigem Migrationshintergrund teilgenommen haben, während der ungewichtete Anteil in der KiGGS-Basiserhebung noch bei $14,7 \%$ lag.

In KiGGS Welle 1 haben außerdem 8,3\% der Teilnehmenden (gewichtet) einen einseitigen Migrationshintergrund (ungewichtet: 7,4\%) (Mikrozensus 2009: $11,0 \%)$. Der prozentuale Anteil an Kindern und Jugendlichen mit einseitigem Migrationshintergrund ist vergleichbar zur Basiserhebung (gewichtet: 8,3\%; ungewichtet: 7,4\%), aber etwas niedriger als im Mikrozensus (• Tab. 3).

Ein Vergleich des gewichteten Anteils der 0- bis 6-Jährigen (Querschnitt) mit den 7- bis 17-Jährigen (Längsschnitt) mit 
Tab. 2 Frauen und Männer mit Migrationshintergrund in DEGS1 und im Mikrozensus 2009 nach Gesundheitsvariablen

\begin{tabular}{|c|c|c|c|c|}
\hline & \multicolumn{2}{|c|}{ Frauen mit Migrationshintergrund } & \multicolumn{2}{|c|}{ Männer mit Migrationshintergrund } \\
\hline & DEGS1 $1_{\text {gewichtet }}$ & Mikrozensus 2009 & DEGS1 $1_{\text {gewichtet }}$ & Mikrozensus 2009 \\
\hline & & $(n=6.570 .000)$ & & $(n=6.460 .000)$ \\
\hline & $\%(95 \%-K I)$ & $\%$ & $\%(95 \%-K I)$ & $\%$ \\
\hline Rauchen & $(p<0,0001)^{*}$ & & $(p=0,0124)$ & \\
\hline Raucherinnen/Raucher & $30,9(26,5-35,6)$ & 22,3 & $41,6(36,2-47,2)$ & 37,3 \\
\hline Nicht-Raucherinnen/Nicht-Raucher & $69,1(64,4-73,5)$ & 77,3 & $58,4(52,8-63,8)$ & 62,7 \\
\hline Body-Mass-Index & $(p=0,2158)$ & & $(p<0,0001)^{*}$ & \\
\hline Untergewicht & $2,7(1,5-4,8)$ & 3,7 & $1,1(0,3-3,7)$ & 0,6 \\
\hline Normalgewicht & $52,0(46,6-57,2)$ & 53,1 & $37,1(31,7-42,8)$ & 38,3 \\
\hline Übergewicht & $30,3(25,5-35,7)$ & 28,2 & $38,6(33,1-44,5)$ & 45,4 \\
\hline Adipositas & $15,0(12,0-18,7)$ & 15,0 & $23,2(18,4-28,7)$ & 15,7 \\
\hline Schwerbehinderung & $(p=0,0515)$ & & $(p=0,5979)$ & \\
\hline Ja & $4,1(2,7-6,1)$ & 5,5 & $6,5(4,4-9,6)$ & 7,0 \\
\hline Nein & $95,9(93,9-97,3)$ & 94,5 & $93,5(90,4-95,6)$ & 93,0 \\
\hline
\end{tabular}

Tab. 3 Anteil von Kindern und Jugendlichen mit Migrationshintergrund in der KiGGS-Basiserhebung, in KiGGS Welle 1 und im Mikrozensus 2009

\begin{tabular}{|c|c|c|c|c|c|c|c|c|c|}
\hline & \multicolumn{3}{|c|}{ Beidseitiger Migrationshintergrund } & \multicolumn{3}{|c|}{ Einseitiger Migrationshintergrund } & \multicolumn{3}{|c|}{ Ohne Migrationshintergrund } \\
\hline & $\mathrm{N}_{\text {ungewichtet }}$ & $\begin{array}{l}\%(95 \% \\
\mathrm{KI})_{\text {ungewichtet }}\end{array}$ & $\begin{array}{l}\%(95 \% \\
\mathrm{KI})_{\text {gewichtet }}\end{array}$ & $\mathrm{N}_{\text {ungewichtet }}$ & $\begin{array}{l}\%(95 \% \\
\mathrm{KI})_{\text {ungewichtet }}\end{array}$ & $\begin{array}{l}\%(95 \% \\
\mathrm{KI})_{\text {gewichtet }}\end{array}$ & $\mathrm{N}_{\text {ungewichtet }}$ & $\begin{array}{l}\%(95 \% \\
\mathrm{KI})_{\text {ungewichtet }}\end{array}$ & $\begin{array}{l}\%(95 \% \\
\mathrm{KI})_{\text {gewichtet }}\end{array}$ \\
\hline $\begin{array}{l}\text { KiGGS-Basiserhebung } \\
(2003-2006)\end{array}$ & 2590 & $\begin{array}{l}14,7 \\
(12,9-16,9)\end{array}$ & $\begin{array}{l}17,6 \\
(15,6-19,9)\end{array}$ & 1292 & $\begin{array}{l}7,4 \\
(6,7-8,1)\end{array}$ & $\begin{array}{l}8,3 \\
(7,6-9,1)\end{array}$ & 13.678 & $\begin{array}{l}77,9 \\
(75,3-80,3)\end{array}$ & $\begin{array}{l}74,1 \\
(71,4-76,6)\end{array}$ \\
\hline $\begin{array}{l}\text { KiGGS Welle } 1 \\
(2009-2012)\end{array}$ & 1104 & $\begin{array}{l}8,9 \\
(7,7-10,3)\end{array}$ & $\begin{array}{l}16,0 \\
(14,0-18,2)\end{array}$ & 917 & $\begin{array}{l}7,4 \\
(6,6-8,3)\end{array}$ & $\begin{array}{l}8,3 \\
(7,5-9,3)\end{array}$ & 10.339 & $\begin{array}{l}83,6 \\
(81,6-85,5)\end{array}$ & $\begin{array}{l}75,7 \\
(73,0-78,2)\end{array}$ \\
\hline $\begin{array}{l}\text { Querschnittproband } \\
\text { (0-6 Jahre) }\end{array}$ & 299 & $\begin{array}{l}6,7 \\
(5,6-8,1)\end{array}$ & $\begin{array}{l}12,8 \\
(10,6-15,3)\end{array}$ & 409 & $\begin{array}{l}9,2 \\
(8,0-10,5)\end{array}$ & $\begin{array}{l}10,0 \\
(8,6-11,6)\end{array}$ & 3742 & $\begin{array}{l}84,1 \\
(81,9-86,1)\end{array}$ & $\begin{array}{l}77,2 \\
(74,0-80,2)\end{array}$ \\
\hline $\begin{array}{l}\text { Längsschnittproband } \\
\text { (7-17 Jahre) }\end{array}$ & 805 & $\begin{array}{l}10,2 \\
(8,8-11,7)\end{array}$ & $\begin{array}{l}17,7 \\
(15,4-20,2)\end{array}$ & 508 & $\begin{array}{l}6,4 \\
(5,7-7,2)\end{array}$ & $\begin{array}{l}7,5 \\
(6,6-8,4)\end{array}$ & 6597 & $\begin{array}{l}83,4 \\
(81,3-85,3)\end{array}$ & $\begin{array}{l}74,9 \\
(72,0-77,5)\end{array}$ \\
\hline Mikrozensus 2009 & 20.289 & & 18,1 & 12.335 & & 11,0 & 79.773 & & 71,0 \\
\hline
\end{tabular}

Migrationshintergrund in KiGGS Welle 1 zeigt, dass der Anteil an Kindern und Jugendlichen mit beidseitigem Migrationshintergrund bei den Jüngeren (Querschnitt) geringer ist (12,8\% vs. $17,7 \%)$. Der Anteil an Kindern und Jugendlichen mit einseitigem Migrationshintergrund ist hier jedoch höher im Vergleich zu den Älteren (Längsschnitt) (10,0\% vs. 7,5\%) (• Tab. 3). Kinder und Jugendliche mit Migrationshintergrund haben insgesamt seltener wieder an KiGGS teilgenommen als Kinder und Jugendliche ohne Migrationshintergrund. Von denjenigen, die sich ein zweites Mal an der KiGGS-Studie beteiligt haben, hatte nur $12,5 \%$ einen Migrationshintergrund, während es in der KiGGS-Basiserhebung 27,1\% waren (Daten nicht gezeigt).

Die Überprüfung der Variablen Alter der Teilnehmenden und Bildungsstand der Eltern (gewichtete Daten) ergab, dass die Stichprobe der Teilnehmenden mit
Migrationshintergrund in KiGGS Welle 1 vor allem hinsichtlich der Bildung gegenüber der KiGGS-Basiserhebung und noch deutlicher gegenüber dem Mikrozensus 2009 verzerrt ist. Die Eltern der Studienteilnehmenden sind zu einem deutlich höheren Anteil besser gebildet, als laut Mikrozensus zu erwarten gewesen wäre. Auch die Altersverteilung der Kinder und Jugendlichen mit Migrationshintergrund unterscheidet sich in einzelnen Altersgruppen von der des Mikrozensus: Bei den Teilnehmenden mit beidseitigem Migrationshintergrund ist die Beteiligung bei den 0- bis 6-Jährigen signifikant geringer im Vergleich zum Mikrozensus. Bei den älteren Kindern und Jugendlichen ist sie hingegen deutlich (und in einigen Altersgruppen signifikant) höher (• Tab. 4).

\section{Diskussion}

\section{Hauptergebnisse}

Menschen mit Migrationshintergrund nehmen offenbar nur dann entsprechend ihrem Anteil an der Bevölkerung an Surveys teil, wenn besondere Anstrengungen bei der Rekrutierung unternommen werden. Damit war es in der KiGGS-Basiserhebung gelungen, Familien mit Migrationshintergrund in repräsentativem Umfang einzubeziehen. Hingegen war die Beteiligung von Kindern und Jugendlichen mit Migrationshintergrund an KiGGS Welle 1 geringer als an der KiGGS-Basiserhebung. Bei den Wiederteilnehmenden (7- bis 17-Jährige) war die Teilnahmerate an KiGGS Welle 1 höher als bei den Ersteingeladenen (0- bis 6-Jährige). Hinsichtlich der Bildung zeigt sich eine geringere Teilnahme bei niedrigem Bildungsniveau der Eltern. In DEGS 1, der sich an Erwach- 
Tab. 4 Vergleich von Kindern und Jugendlichen mit Migrationshintergrund nach Altersgruppen und Bildung der Eltern in der KiGGS-Basiserhebung, in KiGGS Welle 1 und im Mikrozensus 2009

\begin{tabular}{|c|c|c|c|c|c|c|c|c|c|}
\hline & \multicolumn{3}{|c|}{ Beidseitiger Migrationshintergrund } & \multicolumn{3}{|c|}{ Einseitiger Migrationshintergrund } & \multicolumn{3}{|c|}{ Ohne Migrationshintergrund } \\
\hline & $\begin{array}{l}\text { KiGGS- } \\
\text { Basiserhebung }\end{array}$ & $\begin{array}{l}\text { KiGGS } \\
\text { Welle } 1\end{array}$ & $\begin{array}{l}\text { Mikrozen- } \\
\text { sus } 2009\end{array}$ & $\begin{array}{l}\text { KiGGS- } \\
\text { Basiserhebung }\end{array}$ & $\begin{array}{l}\text { KiGGS } \\
\text { Welle } 1\end{array}$ & $\begin{array}{l}\text { Mikrozen- } \\
\text { sus } 2009\end{array}$ & $\begin{array}{l}\text { KiGGS- } \\
\text { Basiserhebung }\end{array}$ & $\begin{array}{l}\text { KiGGS } \\
\text { Welle } 1\end{array}$ & $\begin{array}{l}\text { Mikrozen- } \\
\text { sus } 2009\end{array}$ \\
\hline & $\begin{array}{l}\%(95 \% \\
\mathrm{KI})_{\text {gewichtet }}\end{array}$ & $\begin{array}{l}\%(95 \% \\
\mathrm{KI})_{\text {gewichtet }}\end{array}$ & $\%_{\text {gewichtet }}$ & $\begin{array}{l}\%(95 \% \\
\mathrm{KI})_{\text {gewichtet }}\end{array}$ & $\begin{array}{l}\%(95 \% \\
\mathrm{KI})_{\text {gewichtet }}\end{array}$ & $\%_{\text {gewichtet }}$ & $\begin{array}{l}\%(95 \% \\
\mathrm{KI})_{\text {gewichtet }}\end{array}$ & $\begin{array}{l}\%(95 \% \\
\mathrm{KI})_{\text {gewichtet }}\end{array}$ & $\%_{\text {gewichtet }}$ \\
\hline \multicolumn{10}{|l|}{ Alter } \\
\hline 0-2 Jahre & $\begin{array}{l}14,1 \\
(12,7-15,6)\end{array}$ & $\begin{array}{l}10,4 \\
(8,3-12,9)\end{array}$ & 14,9 & $\begin{array}{l}20,1 \\
(17,7-22,6)\end{array}$ & $\begin{array}{l}18,5 \\
(15,8-21,6)\end{array}$ & 18,4 & $\begin{array}{l}13,8 \\
(13,3-14,4)\end{array}$ & $\begin{array}{l}14,7 \\
(13,8-15,6)\end{array}$ & 14,0 \\
\hline 3-6 Jahre & $\begin{array}{l}22,5 \\
(20,8-24,3)\end{array}$ & $\begin{array}{l}17,8 \\
(15,0-21,1)\end{array}$ & 21,5 & $\begin{array}{l}24,3 \\
(21,6-27,1)\end{array}$ & $\begin{array}{l}23,6 \\
(20,3-27,2)\end{array}$ & 23,8 & $\begin{array}{l}20,1 \\
(19,6-20,7)\end{array}$ & $\begin{array}{l}21,3 \\
(20,2-22,3)\end{array}$ & 20,0 \\
\hline 7-10 Jahre & $\begin{array}{l}22,2 \\
(20,7-23,7)\end{array}$ & $\begin{array}{l}23,2 \\
(20,6-26,1)\end{array}$ & 22,6 & $\begin{array}{l}21,5 \\
(19,4-23,8)\end{array}$ & $\begin{array}{l}24,2 \\
(20,6-28,3)\end{array}$ & 21,9 & $\begin{array}{l}22,4 \\
(21,9-22,9)\end{array}$ & $\begin{array}{l}22,0 \\
(21,0-23,0)\end{array}$ & 22,4 \\
\hline 11-13 Jahre & $\begin{array}{l}18,1 \\
(16,5-19,7)\end{array}$ & $\begin{array}{l}21,2 \\
(18,5-24,3)\end{array}$ & 17,4 & $\begin{array}{l}15,5 \\
(13,6-17,6)\end{array}$ & $\begin{array}{l}15,4 \\
(12,7-18,6)\end{array}$ & 15,6 & $\begin{array}{l}18,4 \\
(17,9-19,0)\end{array}$ & $\begin{array}{l}17,7 \\
(16,9-18,6)\end{array}$ & 18,0 \\
\hline 14-17 Jahre & $\begin{array}{l}23,2 \\
(21,6-24,8)\end{array}$ & $\begin{array}{l}27,4 \\
(24,4-30,6)\end{array}$ & 23,5 & $\begin{array}{l}18,7 \\
(16,0-21,7)\end{array}$ & $\begin{array}{l}18,2 \\
(15,3-21,6)\end{array}$ & 20,2 & $\begin{array}{l}25,2 \\
(24,5-25,9)\end{array}$ & $\begin{array}{l}24,4 \\
(23,3-25,5)\end{array}$ & 25,5 \\
\hline \multicolumn{10}{|c|}{ Bildung Vater ${ }^{a}$} \\
\hline Niedrig & $\begin{array}{l}39,2 \\
(36,9-41,6)\end{array}$ & $\begin{array}{l}33,6 \\
(28,9-38,6)\end{array}$ & 39,0 & $\begin{array}{l}13,9 \\
(11,6-16,6)\end{array}$ & $\begin{array}{l}9,2 \\
(6,6-12,7)\end{array}$ & 16,4 & $\begin{array}{l}6,4 \\
(5,8-7,1)\end{array}$ & $\begin{array}{l}3,7 \\
(2,9-4,6)\end{array}$ & 5,1 \\
\hline Mittel & $\begin{array}{l}42,5 \\
(40,2-44,8)\end{array}$ & $\begin{array}{l}42,7 \\
(38,3-47,3)\end{array}$ & 44,7 & $\begin{array}{l}50,7 \\
(47,1-54,3)\end{array}$ & $\begin{array}{l}45,6 \\
(40,9-50,4)\end{array}$ & 48,4 & $\begin{array}{l}57,8 \\
(56,0-59,6)\end{array}$ & $\begin{array}{l}53,4 \\
(51,2-55,6) \\
\end{array}$ & 56,1 \\
\hline Hoch & $\begin{array}{l}18,3 \\
(16,5-20,2)\end{array}$ & $\begin{array}{l}23,7 \\
(20,5-27,1)\end{array}$ & 15,8 & $\begin{array}{l}35,4 \\
(32,0-38,8)\end{array}$ & $\begin{array}{l}45,2 \\
(40,3-50,3)\end{array}$ & 34,7 & $\begin{array}{l}35,8 \\
(34,0-37,6) \\
\end{array}$ & $\begin{array}{l}42,9 \\
(40,7-45,1)\end{array}$ & 38,6 \\
\hline $\begin{array}{l}\text { nicht be- } \\
\text { stimmbar }\end{array}$ & & & 0,4 & & & 0,5 & & & 0,2 \\
\hline \multicolumn{10}{|c|}{ Bildung Mutter ${ }^{a}$} \\
\hline Niedrig & $\begin{array}{l}49,5 \\
(46,4-52,5)\end{array}$ & $\begin{array}{l}45,0 \\
(40,7-49,4) \\
\end{array}$ & 51,1 & $\begin{array}{l}19,8 \\
(16,9-23,0) \\
\end{array}$ & $\begin{array}{l}16,4 \\
(12,3-21,5) \\
\end{array}$ & 26,9 & $\begin{array}{l}10,9 \\
(9,9-12,0) \\
\end{array}$ & $\begin{array}{l}7,8 \\
(6,6-9,3)\end{array}$ & 10,6 \\
\hline Mittel & $\begin{array}{l}34,7 \\
(32,3-37,2)\end{array}$ & $\begin{array}{l}38,2 \\
(34,5-42,0)\end{array}$ & 36,1 & $\begin{array}{l}57,2 \\
(53,8-60,6)\end{array}$ & $\begin{array}{l}50,9 \\
(46,2-55,6)\end{array}$ & 50,7 & $\begin{array}{l}66,2 \\
(64,7-67,8)\end{array}$ & $\begin{array}{l}64,0 \\
(61,8-66,1)\end{array}$ & 65,4 \\
\hline Hoch & $\begin{array}{l}15,8 \\
(13,9-17,9)\end{array}$ & $\begin{array}{l}16,8 \\
(14,6-19,2)\end{array}$ & 12,4 & $\begin{array}{l}23,0 \\
(20,0-26,2)\end{array}$ & $\begin{array}{l}32,7 \\
(28,4-37,2)\end{array}$ & 22,1 & $\begin{array}{l}22,8 \\
(21,3-24,5)\end{array}$ & $\begin{array}{l}28,2 \\
(26,2-30,2)\end{array}$ & 23,7 \\
\hline $\begin{array}{l}\text { Nicht be- } \\
\text { stimmbar }\end{array}$ & & & 0,5 & & & I & & & 0,3 \\
\hline
\end{tabular}

sene richtete, waren bestimmte Subgruppen mit Migrationshintergrund im Survey unterrepräsentiert. Hierzu zählen selbst zugewanderte Frauen und Männer (1. Generation), Frauen und Männer mit türkischer Staatsangehörigkeit sowie Personen mit niedriger Bildung. In einer umfassenden Analyse unter Einbeziehung weiterer soziodemografischer und migrationsspezifischer Variablen aus DEGS 1 zeigten sich auch bei der Aufenthaltsdauer Verzerrungen [28]. In einzelnen Altersgruppen haben sich Menschen mit Migrationshintergrund allerdings auch stärker am Survey beteiligt, als es entsprechend ihrem Anteil an der Bevölkerung zu erwarten gewesen wäre.

Ein Teil der Unterschiede kann durch die jeweiligen Gewichtungsfaktoren für die Gesamtstichprobe ausgeglichen werden. In DEGS 1 betrifft das jedoch nicht die Unterschiede in der Migrationsgeneration (selbst zugewandert vs. in Deutschland geboren; Variable im Gewichtungsfaktor nicht enthalten) [30]. Außerdem wird in DEGS 1 durch die Gewichtung der Anteil junger Männer mit Migrationshintergrund vergrößert, obwohl die Beteiligung bereits ausreichend war. In KiGGS Welle 1 besteht auch nach der Gewichtung eine Verzerrung hinsichtlich der Bildung: Kinder von Eltern mit hohem Bildungsstand haben signifikant häufiger an KiGGS Welle 1 teilgenommen, als es laut Mikrozensus zu erwarten gewesen wäre. Bei der exemplarischen Überprüfung einiger Gesundheitsindikatoren für Erwachsene zeigten sich zum Teil Verzer- rungen im Vergleich zum Mikrozensus, allerdings ohne einen eindeutigen Trend.

\section{Stärken und Schwächen im}

Sampling der beiden Studien und Vergleich mit anderen Studien

Die in DEGS 1 eingesetzten Maßnahmen zur Steigerung der Response von Menschen mit Migrationshintergrund (Oversampling, übersetzte Fragebögen und Einverständniserklärungen) waren nicht ausreichend, um alle Subgruppen repräsentativ einzubinden. Im Vergleich zu den Teilnehmenden am Bundes-Gesundheitssurvey (BGS 98) zeigt sich allerdings eine Verbesserung: Von denjenigen, die bereits am BGS 98 teilgenommen haben, haben lediglich 6,0\% einen Migrationshinter- 
grund (gewichtet: 7,2\%). Unter denjenigen, die neu an DEGS 1 teilgenommen haben, sind es 14,4\% (gewichtet: 19,8\%). In KiGGS Welle 1 wurde eine geringere Beteiligung als in der KiGGS-Basiserhebung beobachtet. Übersetzte Anschreiben und Fragebögen wurden eingesetzt, allerdings konnten weitere Aktivitäten zur Einbindung von Familien mit Migrationshintergrund nicht realisiert werden. Ein weiterer Grund für die geringere Teilnahme in KiGGS Welle 1 war vermutlich die telefonische Form der Befragung.

Die berichteten Schwierigkeiten bei der Einbeziehung von Menschen mit Migrationshintergrund zeigen sich auch in anderen Studien. So erzielten Pilotstudien für die Nationale Kohorte, die sich speziell an Migrantinnen und Migranten richteten, mit einer registerbasierten Rekrutierungsstrategie Teilnahmequoten von 10$21 \%$ [31]. Mit entsprechend hohem Aufwand (und in Situationen, in denen die Empfänglichkeit für gesundheitliche Belange hoch ist, wie etwa in der Schwangerschaft), lassen sich jedoch auch hohe Teilnahmequoten auch unter Menschen mit Migrationshintergrund erzielen. In der Berliner Perinatalstudie lag die Teilnahmequote bei knapp $90 \%$ [32].

Eine niedrige Responserate erhöht zwar die Wahrscheinlichkeit einer Stichprobenverzerrung, allerdings ist es durchaus möglich, dass die erreichten Personen repräsentativ für die Gruppe mit Migrationshintergrund in einer Bevölkerung sind. Detaillierte Analysen hierzu liegen nur für wenige Studien vor. Darin zeigt sich übereinstimmend, dass Personen, die nicht im Land geboren sind (1. Generation) schlechter erreicht werden [11, 33]. Darüber hinaus nehmen Personen aus bestimmten Herkunftsländern seltener teil [11, 33-35]. Für Deutschland trifft das, in Übereinstimmung mit den DEGS 1-Ergebnissen, insbesondere für Menschen mit türkischem Migrationshintergrund $\mathrm{zu}[31,36]$. In einer anderen Studie aus Deutschland zeigte sich analog zu unseren Ergebnissen eine geringere Teilnahme niedrig qualifizierter Ausländerinnen und Ausländer sowie von Personen mit kurzer Aufenthaltsdauer [35]. In dieser Analyse wurden auch die Auswirkungen der Stichprobenverzerrung auf Gesundheitsvariablen untersucht und Unterschiede im Vergleich mit dem Mikrozensus ermittelt. Es zeigte sich ebenfalls kein eindeutiger Trend im Sinne einer zu positiven oder negativen Einschätzung der Gesundheit aufgrund der Studiendaten.

\section{Stärken und Schwächen des gewählten Vorgehens für den Vergleich}

Für den Vergleich der soziodemografischen und Gesundheitsdaten aus den Surveys wurde der Mikrozensus ausgewählt. Als amtliche Repräsentativstatistik über die Bevölkerung in Deutschland liefert er auch Eckdaten über die in Deutschland lebenden Menschen mit Migrationshintergrund [26]. Es bestehen allerdings Unterschiede in der Erhebungsmethodik des Mikrozensus im Vergleich zu den RKIStudien. Zunächst ist die Teilnahme am Mikrozensus für die ausgewählten Haushalte verpflichtend. Damit sind vor allem die soziodemografischen Angaben (u. a. Bildung) aus dem Mikrozensus sehr belastbar (und wurden auch als Grundlage für die Berechnung der Gewichtungsfaktoren verwendet). Die Angaben zur Gesundheit sind im Mikrozensus hingegen freiwillig. Die Antwortquoten sind aber - im Vergleich zu anderen Gesundheitsbefragungen - ebenfalls hoch. Etwa $70 \%$ der Befragten beantworten beispielsweise Fragen zu Körpergröße und Gewicht [37]. Während aus dem Mikrozensus nur Befragungsdaten vorliegen, wurden in DEGS 1 auch körperliche Untersuchungen durchgeführt (u. a. Messung von Körpergröße und Gewicht). Für den Vergleich wurden nur Befragungsdaten (Face-to-Face) verwendet, denn Größe und Gewicht wurden in DEGS 1 zusätzlich abgefragt. Nicht auszuschließen sind allerdings Effekte unterschiedlicher Befragungsarten in den Erhebungen (Mikrozensus: Face-to-Face-Interview, DEGS 1: Face-to-Face-Interview und Fragebogen, KiGGS Welle 1: Telefoninterview).

Ein weiterer Unterschied ist, dass der Mikrozensus als Haushaltsbefragung angelegt ist. Das bedeutet auch, dass die Fragen nicht unbedingt von allen Haushaltsmitgliedern selbst beantwortet werden müssen. Der sogenannte Haushaltsvorstand gibt in diesem Fall Auskunft. Solche „Proxy-Interviews“ liegen für etwa
$25 \%$ bis $30 \%$ der Personen ab 15 Jahren vor [26] und könnten vor allem bei Angaben zu Rauchstatus, Körpergröße und Gewicht zu Verzerrungen führen.

Geringe Deutschkenntnisse von Menschen mit Migrationshintergrund können auch im Mikrozensus eine Teilnahmebarriere sein, weil die Befragung ausschließlich in deutscher Sprache durchgeführt wird. Allerdings gibt es die Möglichkeit, dass Haushaltsmitglieder gegenseitig die Funktion eines Dolmetschers einnehmen können (oder auch Proxy-Interviews geben). Leider liegt vom Statistischen Bundesamt keine Publikation zum Umgang mit Sprachbarrieren bei der Durchführung des Mikrozensus und eventuellen Auswirkungen vor. Trotz dieser Restriktionen ist der Mikrozensus derzeit die wichtigste Informationsquelle für Daten zu Menschen mit Migrationshintergrund in Deutschland.

\section{Bedeutung der Ergebnisse und Implikationen für die Praxis}

Ganz konkret geben die vorgestellten Ergebnisse zur Beteiligung von Kindern, Jugendlichen und Erwachsenen mit Migrationshintergrund an den Gesundheitsstudien des Robert Koch-Instituts Hinweise auf die Eignung dieser Datenquellen für Auswertungen zur Gesundheit. Analysen zur Gesundheit von Kindern, Jugendlichen und Erwachsenen mit Migrationshintergrund sind mit den Daten generell möglich, allerdings sollten sie stratifiziert erfolgen (bzw. Anwendung statistischer Modelle). Je nach Outcome ist abzuwägen, welche der als problematisch angesehenen soziodemografischen Variablen jeweils Bedeutung haben. Für Erwachsene ist beispielsweise eine Stratifizierung nach Migrationsgeneration (selbst zugewandert vs. in Deutschland geboren) unbedingt zu empfehlen [30]. Für Kinder und Jugendliche ist mit den Daten von KiGGS Welle 1 ein Vergleich der Prävalenzen ausgewählter Gesundheitsvariablen bei Teilnehmenden mit und ohne Migrationshintergrund möglich. Die Auswertungen sollten allerdings bildungsstratifiziert oder -kontrolliert erfolgen. Bei seltenen Erkrankungen bzw. Gesundheitsoutcomes ist eine Auswertung der Daten aus beiden Surveys nicht zu emp- 
fehlen. Die Basiserhebung von KiGGS liefert alternativ eine sehr gute Datengrundlage für zahlreiche Analysen zur Gesundheit der Gruppe von Kindern und Jugendlichen mit Migrationshintergrund.

Darüber hinaus unterstreichen die vorgelegten Ergebnisse, dass für eine repräsentative Einbeziehung von Menschen mit Migrationshintergrund in Studien spezielle Anstrengungen erforderlich sind. Die Übersetzung von Anschreiben und Fragebögen reicht offenbar nicht aus. Die sehr gute Beteiligung von Kindern und Jugendlichen mit Migrationshintergrund an der Basiserhebung von KiGGS hat gezeigt, dass ein umfangreiches Konzept zur Rekrutierung dazu beitragen kann, eine repräsentative Stichprobe dieser Bevölkerungsgruppe zu erzielen. Dafür sollten zunächst die Zielgruppen für eine besondere Teilnahmemotivation klar definiert werden, zum Beispiel entsprechend den häufigsten Herkunftsländern von Menschen mit Migrationshintergrund laut Mikrozensus. Danach sollten Strategien zur verstärkten Rekrutierung oder zum Oversampling diskutiert werden. Bei der Studienplanung sollte auch geklärt werden, wie eventuelle Sprachbarrieren überwunden werden können. Schließlich sind genaue Überlegungen zu den Möglichkeiten und Grenzen der statistischen Analyse der gewonnenen Daten erforderlich. Bei diesen Schritten helfen die hier vorgestellten Ergebnisse.

\section{Weitere (Forschungs-)Aufgaben}

Für die Auswertung der aktuell vorliegenden Datensätze könnte die Entwicklung eines speziellen Gewichtungsfaktors für die Stichprobe von Menschen mit Migrationshintergrund nützlich sein. Dieser müsste weitere, migrationsspezifische Variablen berücksichtigen. Damit könnten Prävalenzen für ausgewählte Gesundheitsoutcomes (ohne Stratifizierung) berichtet werden. Außerdem ergeben sich ggf. spezielle und tiefergehende Auswertungsmöglichkeiten der Daten zur Gesundheit von Menschen mit Migrationshintergrund.

Mit Blick auf die Rekrutierung von Teilnehmenden mit Migrationshintergrund ist bereits geplant, in der 2. Welle der KiGGS-Studie wieder mehr Akti- vitäten zur Einbindung von Migrantinnen und Migranten durchzuführen. Unter anderem sollen Multiplikatoren dafür gewonnen werden, die Studie zu unterstützen und zur Teilnahme zu motivieren. KiGGS Welle 2 hat im September 2014 begonnen und wird im Jahr 2016 abgeschlossen.

\section{Fazit}

Menschen mit Migrationshintergrund machen einen substanziellen Anteil der Bevölkerung in Deutschland aus. Sie können gegenüber der Mehrheitsbevölkerung gesundheitlich benachteiligt sein. Dennoch werden sie bislang in Gesundheitssurveys nicht immer einbezogen. Wenn sich Surveys an Menschen mit Migrationshintergrund richten, führen unter anderem sprachliche Barrieren oftmals zu geringen Teilnahmequoten. Die hier vorgestellten Auswertungen zeigen, dass bei Einsatz übersetzter Fragebögen und Anschreiben eine Beteiligung erreicht werden kann, die - mit einigen Restriktionen - statistische Auswertungen der erhobenen Gesundheitsdaten für diese Bevölkerungsgruppe ermöglicht. Hieraus leitet sich auch die Forderung nach einer weiteren Verbesserung der Datenlage zur Gesundheit von Menschen mit Migrationshintergrund $a b$, indem sie in alle neu aufgesetzten Gesundheitsstudien umfassend einbezogen werden sollten. Dazu sind entsprechendes Know-how und natürlich auch zusätzliche Ressourcen nötig. Aufgrund der Bevölkerungsstruktur in Deutschland und deren Entwicklung ist diese Forderung wichtig und wird in Zukunft weiter an Relevanz gewinnen.

\section{Korrespondenzadresse}

Dr. A.-C. Saß

Abteilung für Epidemiologie und

Gesundheitsmonitoring

Robert Koch-Institut

General-Pape-Str. 62-64, 12101 Berlin

SassA@rki.de

\section{Einhaltung ethischer Richtlinien}

Interessenskonflikt. A.-C. Saß, B. Grüne, A.-K. Brettschneider, A. Rommel, O. Razum und U. Ellert erklären, dass kein Interessenskonflikt vorliegt.

\section{Literatur}

1. Statistisches Bundesamt (2014) Bevölkerung mit Migrationshintergrund - Ergebnisse des Mikrozensus 2013. Fachserie 1 Reihe 2.2. Statistisches Bundesamt, Wiesbaden

2. Knipper M, Bilgin Y (2009) Migration und Gesundheit. Konrad-Adenauer-Stiftung e. V., Sankt Augustin, Berlin

3. Razum O, Zeeb H, Meesmann U et al (2008) Migration und Gesundheit. Schwerpunktbericht der Gesundheitsberichterstattung des Bundes. Robert Koch-Institut, Berlin

4. Schenk L, Neuhauser H, Ellert U (2008) Kinder- und Jugendgesundheitssurvey (KiGGS) 2003-2006: Kinder und Jugendliche mit Migrationshintergrund in Deutschland. Beiträge zur Gesundheitsberichterstattung des Bundes. Robert Koch-Institut, Berlin

5. Butler J, Albrecht N-J, Ellsäßer G et al (2007) Migrationssensible Datenerhebung für die Gesundheitsberichterstattung. Bundesgesundheitsbl Gesundheitsforsch Gesundheitssch 50(10):1232-1239

6. Schenk L (2002) Migrantenspezifische Teilnahmebarrieren und Zugangsmöglichkeiten im Kinderund Jugendgesundheitssurvey. Gesundheitswesen 64(S1):59-68

7. Schenk L, Ellert U, Neuhauser H (2007) Kinder und Jugendliche mit Migrationshintergrund in Deutschland: Methodische Aspekte im Kinderund Jugendgesundheitssurvey (KiGGS). Bundesgesundheitsbl Gesundheitsforsch Gesundheitssch 50(5-6):590-599

8. Schenk L, Neuhauser H (2005) Methodische Standards für eine migrantensensible Forschung in der Epidemiologie. Bundesgesundheitsbl Gesundheitsforsch Gesundheitssch 48(3):279-286

9. Razum O, Zeeb H, Akgün S (2001) How useful is a name-based algorithm in health research among Turkish migrants in Germany? Trop Med Int Health 6(8):654-661

10. Ibrahim S, Sidani S (2014) Strategies to recruit minority persons: a systematic review. J Immigr Minor Health 16(5):882-888

11. Denktaş S, Koopmans G, Birnie E et al (2009) Ethnic background and differences in health care use: a national cross-sectional study of native Dutch and immigrant elderly in the Netherlands. Int J Equity Health 8:35

12. Gößwald A, Lange M, Kamtsiuris P et al (2012) DEGS: Studie zur Gesundheit Erwachsener in Deutschland. Bundesweite Quer- und Längsschnittstudie im Rahmen des Gesundheitsmonitorings des Robert Koch-Instituts. Bundesgesundheitsbl Gesundheitsforsch Gesundheitssch 55(67):775-780

13. Kamtsiuris $P$, Lange $M$, Hoffmann R et al (2013) Die erste Welle der Studie zur Gesundheit Erwachsener in Deutschland (DEGS1). Bundesgesundheitsbl Gesundheitsforsch Gesundheitssch 56(5-6):620630

14. Kurth B-M (2012) Das RKI-Gesundheitsmonitoring - was es enthält und wie es genutzt werden kann. Public Health Forum. Elsevier 20:e1-4.e3 


\section{Leitthema}

15. Kurth B-M, Lange C, Kamtsiuris P et al (2009) Gesundheitsmonitoring am Robert Koch-Institut. Bundesgesundheitsbl Gesundheitsforsch Gesundheitssch 52(5):557-570

16. Scheidt-Nave C, Kamtsiuris P, Gosswald A et al (2012) German health interview and examination survey for adults (DEGS) - design, objectives and implementation of the first data collection wave. BMC Public Health 12:730

17. Hölling H, Schlack R, Kamtsiuris P et al (2012) Die KiGGS-Studie. Bundesweit repräsentative Längsund Querschnittstudie zur Gesundheit von Kindern und Jugendlichen im Rahmen des Gesundheitsmonitorings am Robert Koch-Institut. Bundesgesundheitsbl Gesundheitsforsch Gesundheitssch 55(6-7):836-842

18. Kamtsiuris $P$, Lange M, Schaffrath Rosario $A$ (2007) Der Kinder- und Jugendgesundheitssurvey (KiGGS): Stichprobendesign, Response und Nonresponse-Analyse. Bundesgesundheitsbl Gesundheitsforsch Gesundheitssch 50(5-6):547-556

19. Kurth BM, Kamtsiuris $P$, Hölling $\mathrm{H}$ et al (2008) The challenge of comprehensively mapping children's health in a nation-wide health survey: design and first results of the German KiGGS-Study. BioMed Cent Public Health 8:196

20. Gößwald A, Lange M, Dölle R et al (2013) Die erste Welle der Studie zur Gesundheit Erwachsener in Deutschland (DEGS1). Bundesgesundheitsbl Gesundheitsforsch Gesundheitssch 56(5-6):611-619

21. Robert Koch-Institut (2009) DEGS-Studie zur Gesundheit Erwachsener in Deutschland. Projektbeschreibung. Beiträge zur Gesundheitsberichterstattung des Bundes. Robert Koch-Institut, Berlin

22. Lange M, Butschalowsky HG, Jentsch F et al (2014) Die erste KiGGS-Folgebefragung (KiGGS Welle 1): Studiendurchführung, Stichprobendesign und Response. Bundesgesundheitsbl Gesundheitsforsch Gesundheitssch 57(7):747-761

23. Robert Koch-Institut (RKI) (2011) KiGGS - Kinderund Jugendgesundheitsstudie Welle 1 - Projektbeschreibung. Robert Koch-Institut, Berlin

24. Thefeld W, Stolzenberg H, Bellach B-M (1999) Bundes-Gesundheitssurvey: Response, Zusammensetzung der Teilnehmer und Non-Responder-Analyse. Gesundheitswesen 61(2):S57-S61

25. Robert Koch-Institut (RKI) (2008) Kinder- und Jugendgesundheitssurvey (KiGGS) 2003-2006: Kinder und Jugendliche mit Migrationshintergrund in Deutschland. Robert Koch-Institut, Berlin

26. Statistisches Bundesamt (2014) Erläuterungen zum Mikrozensus https://www.destatis.de/DE/ ZahlenFakten/GesellschaftStaat/Bevoelkerung/ Methoden/Mikrozensus.html (Destatis) Zugegriffen: 07. Nov. 2014

27. Statistisches Bundesamt (2010) Mikrozensus 2009: Qualitätsbericht. Statistisches Bundesamt, Wiesbaden

28. Grüne B (2013) Beteiligung von Menschen mit Migrationshintergrund an der ersten Welle der Studie zur Gesundheit Erwachsener in Deutschland (DEGS 1). Fakultät für Gesundheitswissenschaften, Universität Bielefeld, Bielefeld

29. Schroedter JH, Lechert $Y$, Lüttinger $P$ (2006) Die Umsetzung der Bildungsskala ISCED-1997 für die Volkszählung 1970, die Mikrozensus-Zusatzerhebung 1971 und die Mikrozensen 1976-2004. ZUMA-Methodenbericht. ZUMA, Mannheim
30. Rommel A, Saß A, Born S et al (2015) Die gesundheitliche Lage von Menschen mit Migrationshintergrund und der Einfluss des sozioökonomischen Status. Erste Ergebnisse der Studie zur Gesundheit Erwachsener in Deutschland (DEGS1). Bundesgesundheitsbl Gesundheitsforsch Gesundheitssch 58(5):XX-XX

31. Reiss K, Dragano N, Ellert U et al (2014) Comparing sampling strategies to recruit migrants for an epidemiological study. Results from a German feasibility study. Eur J Public Health. doi:10.1093/eurpub/ cku046

32. Brenne S, David M, Borde T et al (2015) Werden Frauen mit Migrationshintergrund von den Gesundheitsdiensten gleich gut erreicht? Das Beispiel Schwangerenvorsorge in Berlin. Bundesgesundheitsbl Gesundheitsforsch Gesundheitssch 58(5):XX-XX

33. Carlsson F, Merlo J, Lindstrom M et al (2006) Representativity of a postal public health questionnaire survey in Sweden, with special reference to ethnic differences in participation. Scand J Public Health 34(2):132-139

34. Moradi T, Sidorchuk A, Hallqvist J (2010) Translation of questionnaire increases the response rate in immigrants: filling the language gap or feeling of inclusion? Scand J Public Health 38(8):889-892

35. Schenk L, Neuhauser $H$ (2005) Beteiligung von Migranten im telefonischen Gesundheitssurvey: Möglichkeiten und Grenzen. Gesundheitswesen 67(10):719-725

36. Winkler V, Leitzmann M, Obi N et al (2014) Response in individuals with and without foreign background and application to the National Cohort in Germany: which factors have an effect? Int J Public Health 59(3):555-563

37. Statistisches Bundesamt (2014) Mikrozensus 2013- Fragen zur Gesundheit. Körpermaße der Bevölkerung. Statistisches Bundesamt, Wiesbaden 